\title{
A MASS PRODUCTION OF THICK-FILM CAR VOLTAGE REGULATORS
}

\author{
D. A. BIANCHI, P. FARAVELLI and G. S. RODARI \\ Magneti Marelli, Divisione Sistemi ed Elettronica, 27100 Pavia, Italy
}

(Received August 31, 1977)

\begin{abstract}
The technological solutions chosen for a car-voltage regulator now in mass production (thick-film hybrid with standard encapsulated active devices) are discussed from the point of view of functional performance, producibility and reliability.
\end{abstract}

\section{INTRODUCTION}

The electric generator on cars must be regulated to guarantee a constant output voltage against changes in load and motor speed. Also the temperature dependence of this voltage must be considered for matching problems with the battery.

Traditionally this was accomplished by controlling the excitation current through relays of the vibratingcontact type, with a bimetallic strip acting as a load spring to introduce the desired temperature compensation.

The continuous efforts by car manufacturers to minimize maintenance operations has focused attention on the inherent limitations of this system (arcing on contacts, sensitivity to vibrations, poor regulation and temperature compensation, shifts with time of the regulated voltage) as a source of trouble, both directly (failure of the device) and indirectly (effect on the battery of a poor matching with generator voltage).

An electronic regulator had to be introduced to achieve tighter tolerances, better power-handling capability and higher reliability.

\section{A BASIC TECHNOLOGICAL CHOICE}

To implement electronic systems for automotive applications thick-film technology has been chosen on the basis of the following considerations.

\subsection{Reliability}

The automotive environment can be very tough, with ambient operating temperatures from -40 to $100^{\circ} \mathrm{C}$ and above. Frequent changes in ambient temperature plus intermittent operation result in severe thermal cycling. There may be strong vibrations and mechanical abuse by unskilled people, physicalchemical actions induced by solvents, acids, water, salts, etc.

The strong and compact structure of thick-film modules, their good thermal properties, the better reproducibility and decrease in solder joints afforded by in tegration techniques are all factors in achieving the low failure rates car manufacturers expect from electronic devices.

\subsection{Miniaturization}

Space and weight requirements are often critical in today's crowded cars, and the reduced dimensions made possible by thick-film integration are a definite advantage.

Another positive result of this is the possibility of using better resins for encapsulation even though they are more expensive.

\subsection{Functional Trimming}

Functional trimming of thick-film resistors to compensate for the spread in component characteristics is a low-cost operation on automatic laser systems. Use of discrete potentiometers is more costly and less reliable.

\section{PRODUCTION ORGANIZATION}

In the production of hybrid modules the main choices 
bear on two areas:

1) Selection of materials, and especially of components (encapsulated devices or semiconductor chips).

2) Optimization of methods and of degree of automation.

It has been decided to group together in a single controlled-atmosphere room all operations pertaining to thick-film technology, for all types of circuits. Automatic screen-printers are used and the substrates, usually with more than one circuit per piece, are automatically unloaded on $12 \mathrm{in}$. belt furnaces. Single circuits are separated by laser scribing, using a digitally-controlled $\mathrm{CO}_{2}$-system of our own manufacturing. Resistors are trimmed on automatic YAG-laser systems controlled by minicomputers.

Encapsulated active devices and chip capacitors are used for the regulator, with manual placement on screen-printed solder pads and reflow-soldering in a belt furnace.

Semi-automatic machines are then used to mount the circuits in aluminum heat-sinks using an insulating resin of high thermal conductivity; to encapsulate it with another resin and to complete the mechanical assembly.

After thermal cycling the regulators are tested
$100 \%$ on a fully automatic machine, both at ambient temperature and at $100^{\circ} \mathrm{C}$.

\section{RESULTS AND DISCUSSION}

The use in this device of encapsulated active components instead of dice resulted, after lengthy examinations, from the following considerations:

a) In this particular device the increase in miniaturization to be gained with chips is not great (see Figure 1) and is not important for practical purposes.

b) It was felt that the difference in price for the components would actually be offset by:

- yield decrease (both because the chip cannot be completely tested before being mounted, and because chip mounting is not a batch process and all the single yields must be multiplied together).

- increased costs in equipment and labout both for assembly and quality control (most of these costs are already included in the price of packaged components, which profit from being assembled in very large quantities on automatic lines using wellestablished methods, materials and quality-control procedure).

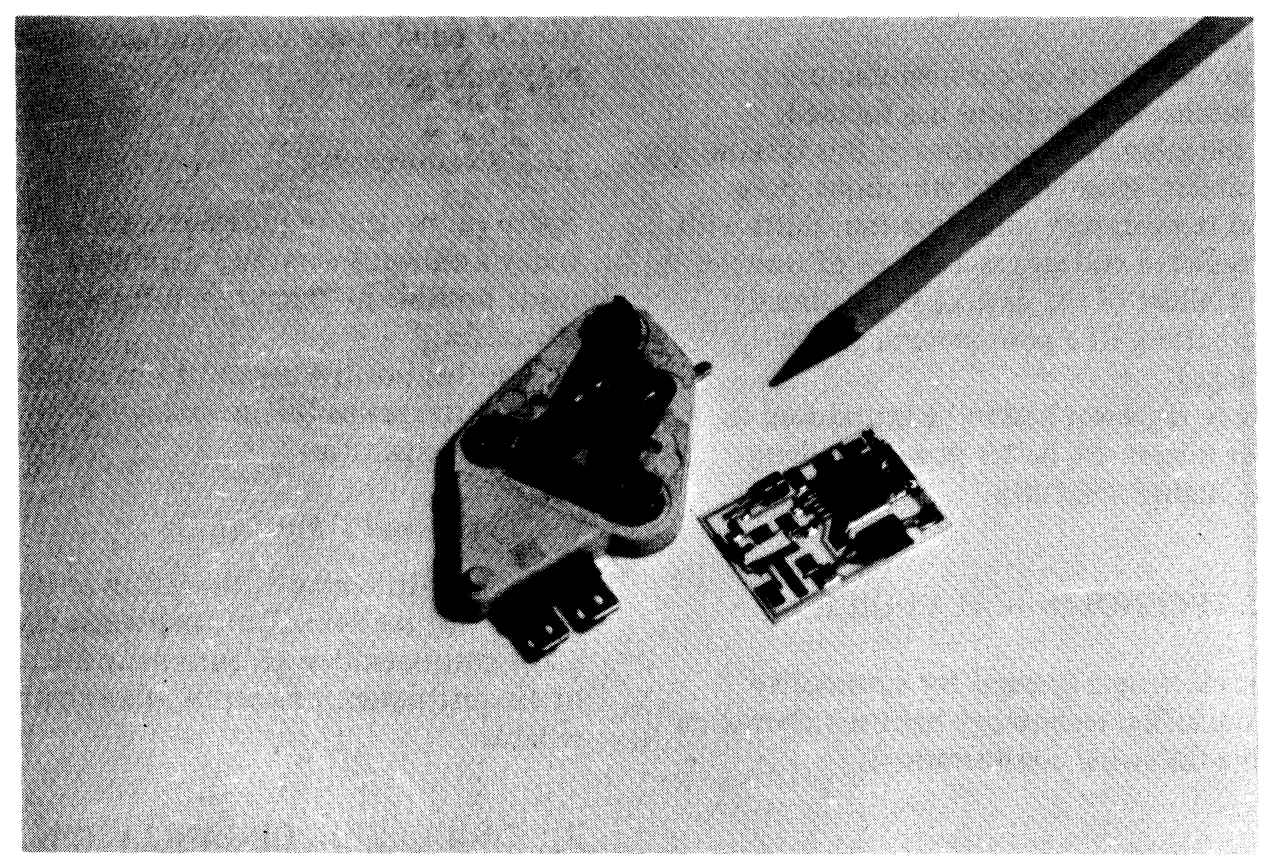

FIGURE 1 Comparison between a U.S. made regulator with dice (left) and circuit with packaged active devices. 


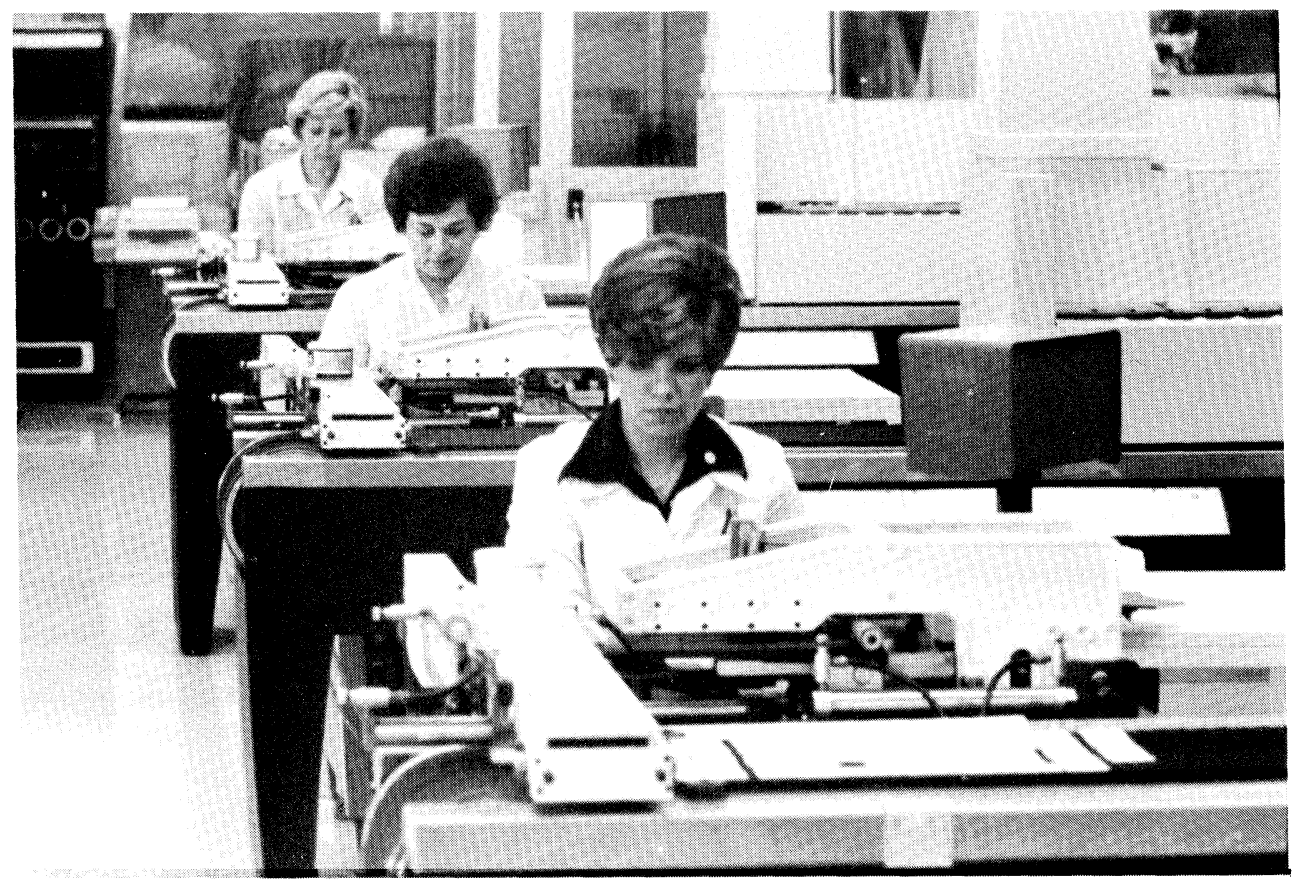

FIGURE 2 View of screen-printing operation.

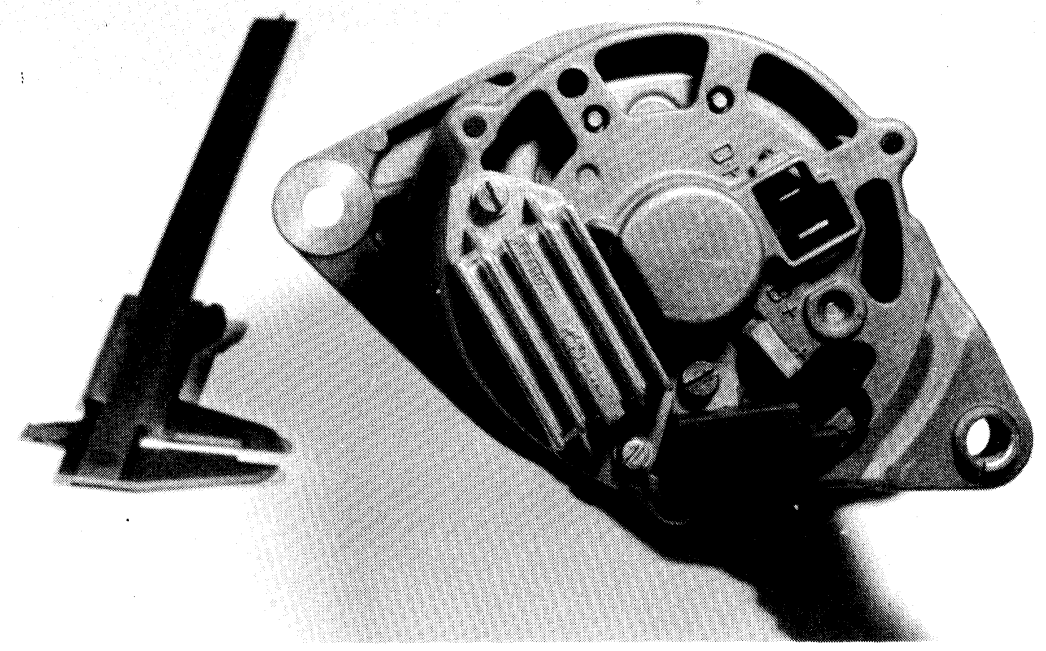

FIGURE 3 Regulator built into the brush-holder assembly. 


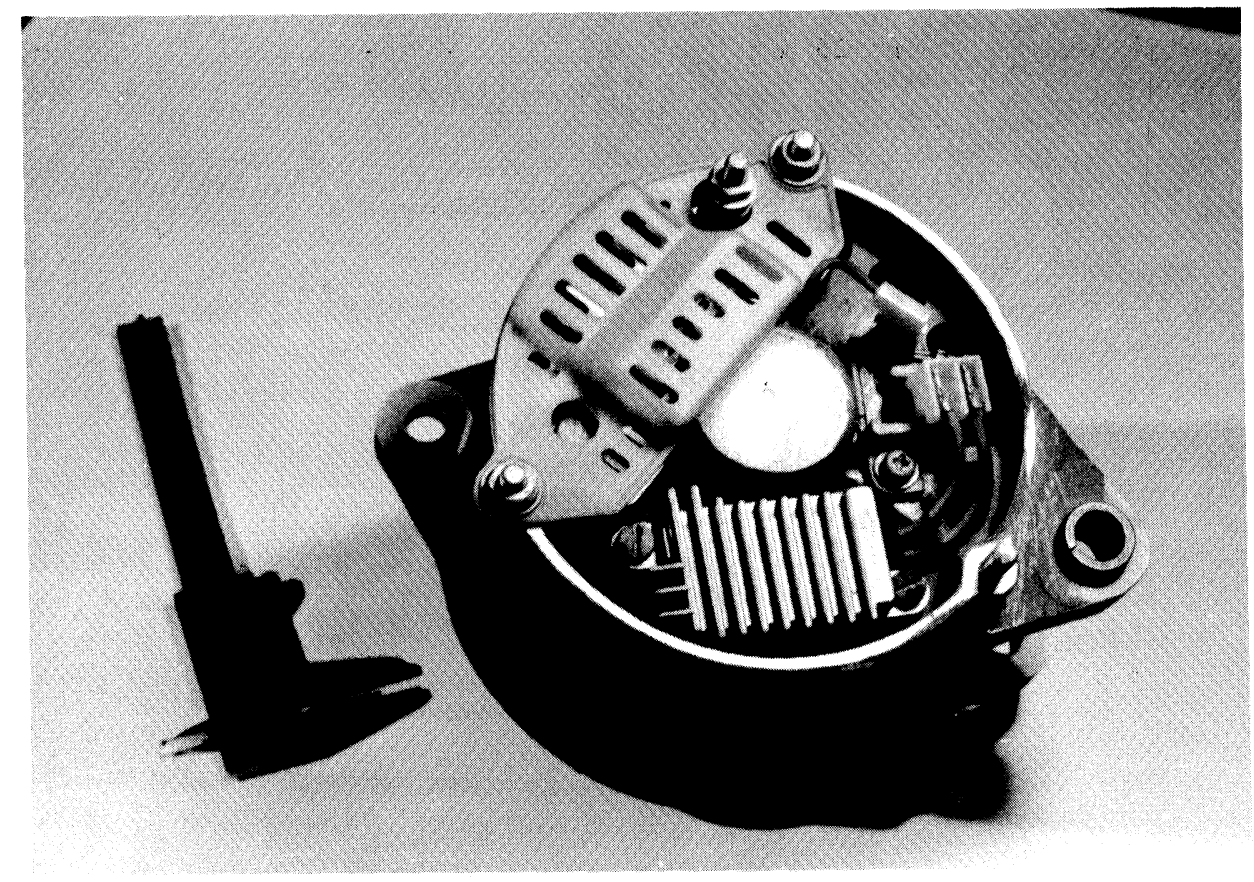

FIGURE 4 Regulator mounted independently on the alternator.

Placing packaged components on solder pads is cheap, and will be automatized when higher volumes are reached by the end of the year. The result of such an analysis may well be the opposite for another product having tighter requirements for miniaturization and/or using different components with a more favourable price difference.

Taking advantage of thick-film technology the regulator is small enough to be built into the alternator, independently or in the brush-holder assembly; a tolerance of $\pm 0.5 \%$ is easily obtained through functional trimming; its sturdy structure and the reproducibility of the process, monitored through proper quality control procedures, have yielded consistently good results in the field $(0.2 \%$ failures during the six-month guarantee period of cars).

Production rate is at present 3000 regulators per day; it is foreseen that by the end of year it will be near 5000 units/day. 

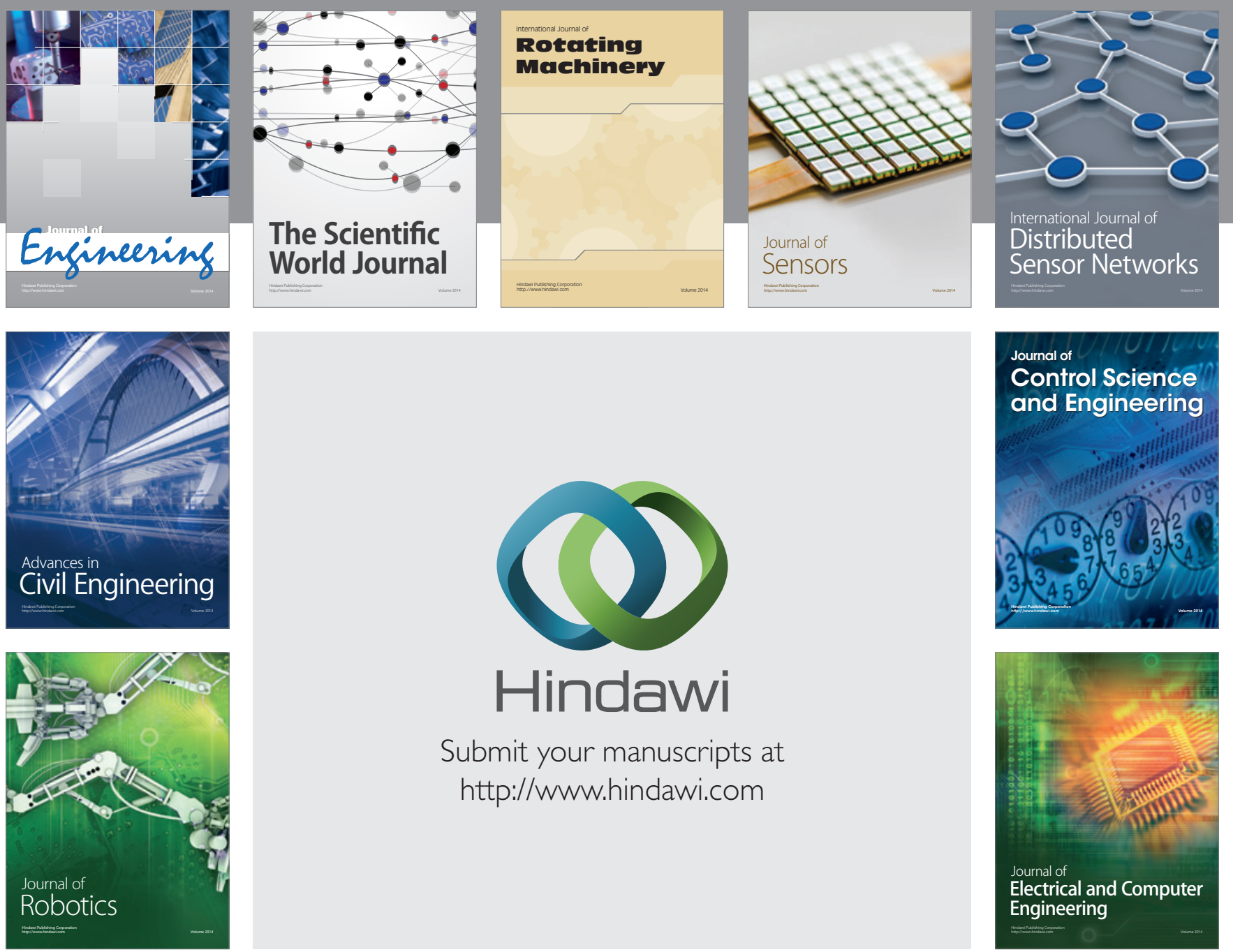

Submit your manuscripts at

http://www.hindawi.com
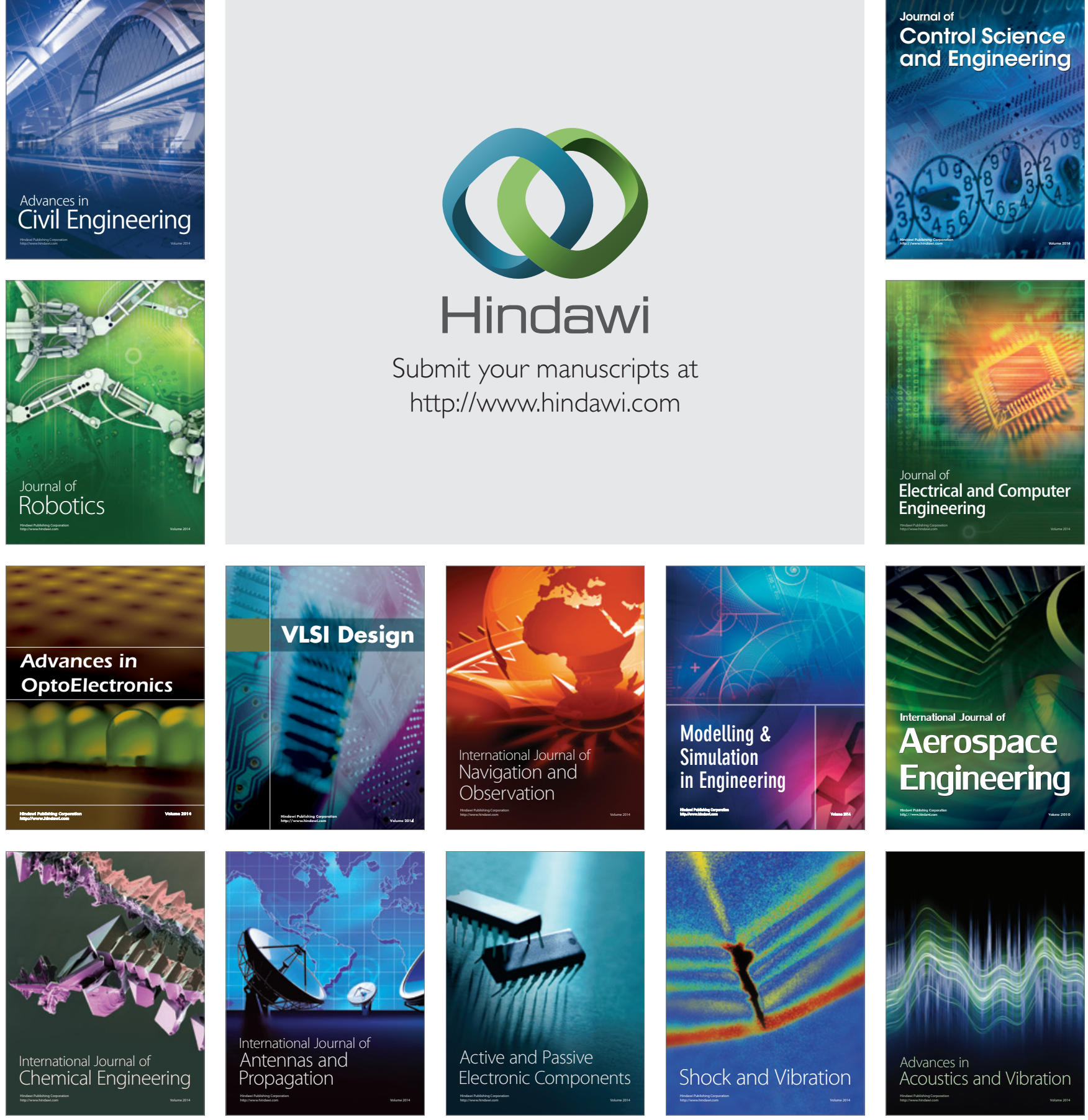